\title{
Acquiring orphans
}

\author{
Rogier Rooswinkel, Geert-Jan Mulder \& Sander van Deventer
}

Will your orphan drug startup draw acquisition interest?

$\mathrm{H}$ istorically, development of drugs for rare diseases has been neglected because of a perceived lack of return on investment. This began to change when the Orphan Drug Act (ODA) passed in the United States in 1983, providing companies with incentives to develop drugs for rare diseases, including tax credits on trial costs, a waiver of the U.S. Food and Drug Administration (FDA) fee, grants for clinical trials, additional protocol assistance and seven years of market exclusivity. Similar rules have since been adopted in Europe, Japan and other regions.

The ODA generally has been considered successful. Since its acceptance, more than 2,800 orphan designations have been assigned and more than 400 products have received market authorization (http://www.accessdata.fda.gov/scripts/opdlisting/oopd/). But there are a few other factors that positively influence the economics of orphan drug development: timelines are typically shorter ${ }^{1}$; the FDA is often more flexible with approvals because of the lack of alternative treatments ${ }^{2}$; and approved orphan drugs often require less marketing, have a faster uptake and are generally well reimbursed ${ }^{1}$.

All these considerations have made orphan drug development strategies increasingly popular with big pharma and venture capital investors-and, thus, with biotech entrepreneurs. But what financial rewards have startup orphan drug companies actually reaped, and how likely is it that your company will draw interest from big-pocketed buyers?

We set out to answer these questions and examined the acquisition characteristics of

Rogier Rooswinkel is Analyst and

Geert-Jan Mulder and Sander van Deventer are Partners at Forbion Capital Partners, Naarden,

The Netherlands. Sander van Deventer is

Professor at Leiden University Medical Center,

Leiden, The Netherlands.

e-mail:Sander.van.Deventer@forbion.com companies both with and without a rare disease focus. We were able to identify the important acquirers, average price of purchase and at what stage orphan disease companies often get bought.

This information should help inform bioentrepreneurs about the orphan drug space and their potential futures in it.

\section{Timelines}

Our methodology (Box 1) gave us 98 mergers and acquisitions (M\&A) in 2008-2012 involving startup drug development companies. Of these, $28 \%$ had an orphan designation (ODN) on the lead compound and another $13 \%$ had an ODN on a product other than its lead compound (Fig. 1). Hence, more than $40 \%$ of the acquired biotech companies had an ODN compound in development. It would require data on the number of orphan-designated drugs in development as a percentage of all drugs in pipelines to estimate the interest in ODN-focused companies among acquirers, but these data are not available. The next best estimate would be the number of orphandesignated drug approvals as a percentage of

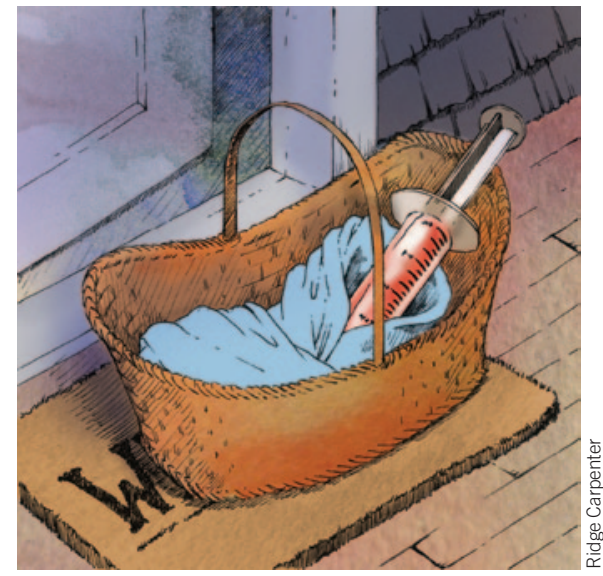

all drug approvals. This hovered around 35\% for $2008-2012^{3}$. As the percentage of orphanfocused acquisitions is in that same range, our data suggest that there is a healthy appetite for companies with a rare disease focus. Next, we set out to investigate differences in the deal parameters related to the ODN status of the lead compound. We extracted the company founding dates from various public sources and the BioCentury Online Intelligence database and calculated the time from company

\section{Box 1 Our methodology}

Our analysis made use of the HBM Partners Mergers and Acquisitions Report, which tracks all biotech acquisitions of both public and private companies based in the United States and Europe ${ }^{8}$. We identified which of the companies in the report had an orphan focus (by having an orphan designation from either the U.S. Food and Drug Administration or the European Medicines Agency on the lead compound; in the vast majority of the deals, the lead compound was the value driver of the deal) and restricted our analysis to the last five years. We curated the database to contain only drug development companies, excluding companies that develop diagnostics or sell over-the-counter or generic medicines or have platform technologies without lead compounds. We also did not incorporate acquisitions of well-established companies, defined as those having more than one marketed product.

On the buyer's side, we restricted our analysis to companies that acquired at least 2 companies in 2008-2012, allowing us to view them as active. This left us with a dataset of 98 acquisitions in the biotech field, for which we further completed and amended the deal data when possible from public sources and the BioCentury Online Intelligence database. 


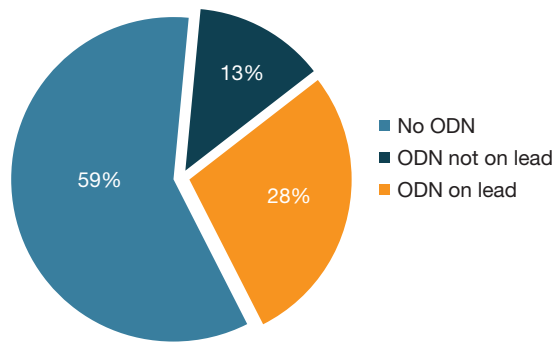

Figure 1 Orphan designation (ODN) status for companies in our dataset.

inception to acquisition (Fig. 2a). Companies without an ODN on their lead compound had a median of eight years from founding to buyout-the same time to acquisition for companies with an orphan focus. However, the majority of non-orphan biotech companies gets acquired at phase 2, whereas orphan drug companies tend to get acquired at a later stage (Fig. 2b); more than $62 \%$ of the companies with an ODN on their lead were acquired at phase 3 or later compared with less than 33\% of non-orphan-based companies. Therefore, although our data show that orphan drug development is indeed faster, it does not mean a shorter time to acquisition. This might be caused by acquirers needing more validation before betting on a compound with a perceived limited market potential and possible difficulties related to patient recruitment. In addition, buyers may need more clinical data to embrace a new method or platform, especially as phase 2 trials for rare diseases often involve a limited number of patients. Alternatively, the later-stage acquisitions may reflect the fact that phase 3 development of many orphan drugs, in contrast to most nonorphan drugs, often have smaller trials and can be privately financed. Bioentrepreneurs might therefore be less inclined to sell their company after the phase 2 trial. Taken together, this information indicates that bioentrepreneurs with an orphan-focused company should keep in mind that acquisition timelines might be as long as for any other biotech; thus, their financing status should allow for the complete development of a compound in-house.

We next investigated whether development of an orphan drug really makes for an attractive financial investment. To get a clear picture on the multiples on investment, we considered only the privately held companies that were directly acquired by big pharma. We observed no significant differences between ODN compound-driven deals and non-ODN deals. This included-but was not limited to-capital invested until exit (Fig. 3a), the total deal value split out into development stages (Fig. 3b) and, importantly, the multiples on total capital invested (Fig. 3c). For instance, the median amount invested before exit was $\$ 60$ million for non-orphan-based companies and \$51 million for companies with an ODN as their lead compound. Also, these deals had a median return on invested capital of $5.8 \times$ for companies with an orphan designation and $5.5 \times$ for those without. Because rare disease-focused companies were acquired at a later stage of clinical development (Fig. 2b), our data support the notion that orphan drug development is less costly ${ }^{1}$. On the other hand, the more advanced development stage is not reflected in total deal value or upfront payment and therefore does not result in higher multiples on the invested capital. In essence, this shows that in the case of an exit, from a purely financial standpoint it is about as attractive to develop drugs for a rare disease as it is for a more common illness. Thus, financial and timeline considerations should not necessarily entice individuals toward, nor keep them away from, developing or investing in drugs for rare diseases. a

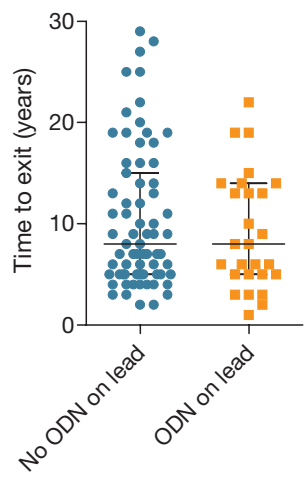

b

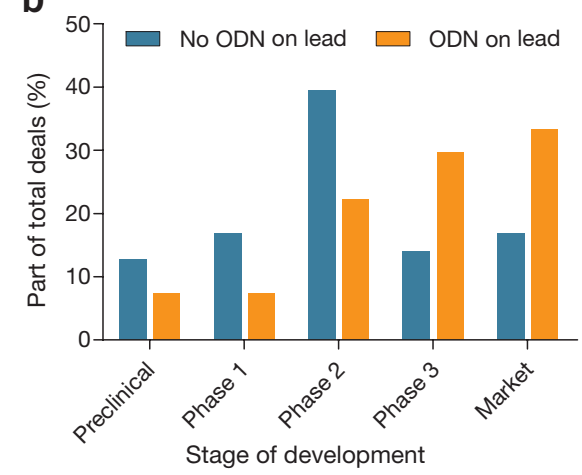

Figure 2 Time to acquisition and phase of development at acquisition. (a) Time from company inception to acquisition. Lines indicate median and interquartile ranges. (b) Phase of development of the lead compound at time of acquisition. Bars indicate number of acquisitions with compounds at that stage as a percentage of the total number of acquisitions. ODN, orphan designation.

Often, after acquiring an ODN, a pharmaceutical company will develop that same drug for a broader indication. Orphan-designated drugs such as erythropoietin and rituximab initially benefited from the ODA incentives and subsequently became blockbusters through label extension. Pharmaceutical
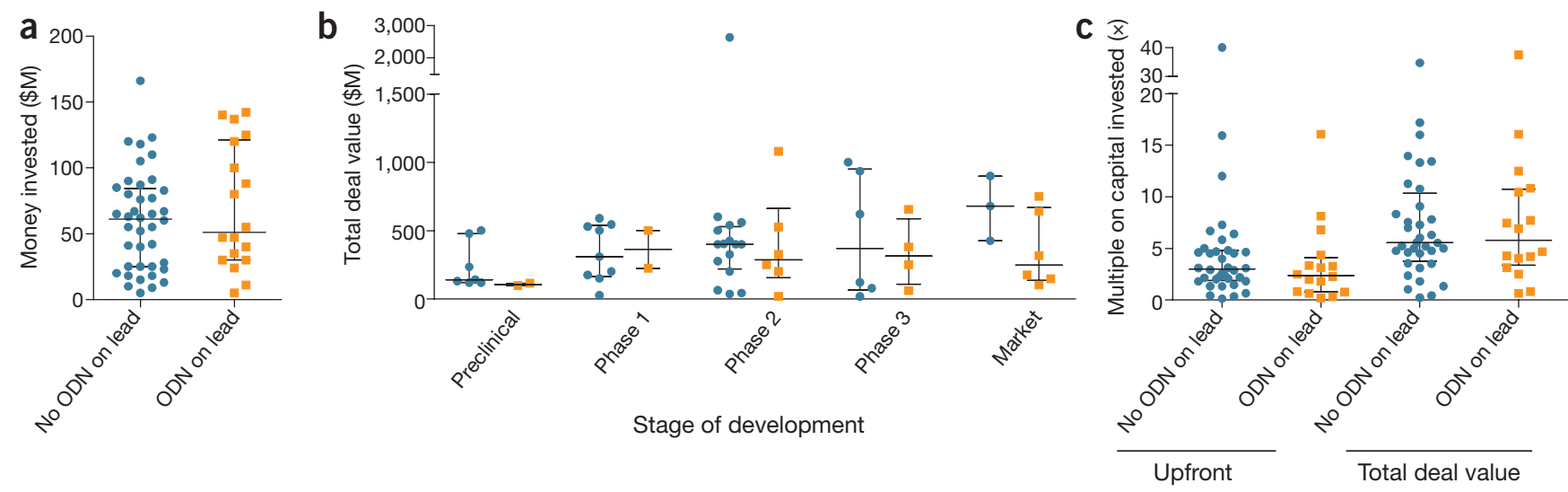

Figure 3 Orphan designation (ODN)- and non-ODN-driven deals. (a-c) Dots indicate the deal parameters of individual companies until the company was acquired. Lines indicate median and interquartile range. 
Table 1 Examples of dedicated and non-dedicated orphan drugs in our dataset

\begin{tabular}{lllllll} 
Company & Compound & Type & Method of action & Orphan designation & Broader indication & Dedicated \\
\hline Kosan & Tanespimycin & Small molecule & Heat shock protein 90 inhibitor & Multiple myeloma, chronic myeloid leukemia & Solid tumors & No \\
Gemin-X & Obatoclax & Small molecule & Bcl-2 family inhibitor & Chronic lymphocytic leukemia & Solid tumors & No \\
FoldRx & Tafamidis & Small molecule & Stabilization of transthyretin & Transthyretin amyloid polyneuropathy & None & Yes \\
\hline Lev & Cinryze & Isolated protein & C1 esterase inhibitor & $\begin{array}{l}\text { Routine prophylaxis of hereditary } \\
\text { angioedema }\end{array}$ & None & Yes \\
Pharmaceuticals & & & & &
\end{tabular}

companies-Novartis in particular-have now fully adopted this approach ${ }^{4}$. This holds especially true in cancer, in which the same molecular pathways often are deregulated in both rare and common cancers.

This interest in oncology is also reflected in the FDA's ODN database. In 2008-2012, 7 of the 10 most prevalent ODNs in the database were in oncology (http://www.accessdata.fda.gov/ scripts/opdlisting/oopd/). This is in line with previous research noting that since the ODA, oncology accounts for $33 \%$ of the total ODNs ${ }^{5}$. To correct for the 'oncology ODN effect', we defined a category of 'dedicated orphan drugs' being specifically developed for one or more related orphan indications and lacking an obvious application in a larger, non-orphan indication, and we divided the deals into dedicated or non-dedicated categories (Table 1). We subsequently reanalyzed deal parameters-time to exit, stage of development and multiples on capital invested-but did not observe any significant differences between drugs with or without an ODN. On the other hand, this 'oncology ODN' strategy may suit small biotechs seeking to develop drugs for larger markets.

\section{Who buys orphan drug programs?}

Rare disease-focused startups and bioentrepreneurs who might be thinking about launching an orphan drug company need to know which pharmaceutical and biotech companies have interest in the orphan space. We identified the companies that received the most FDA-designated ODNs in 2008-2012 and also included their assigned ODNs from the European Medicines Agency (EMA) (http:// ec.europa.eu/health/documents/communityregister/html/orphreg.htm) (Fig. 4a). Novartis is a clear winner in both Europe and the United States, and it also ranks first in terms of orphan drug revenues (http://www. evaluategroup.com/orphandrug2013), followed by GlaxoSmithKline, Roche and Pfizer. Surprisingly, we found a large difference in the number of ODNs with the FDA and EMA for some big pharma companies. For instance, Roche has 15 ODNs with the FDA but none with the EMA.

Based on this analysis, Novartis, GlaxoSmithKline and Roche would appear to be suitable candidates for M\&A discussions, but this is not substantiated by their number of acquisitions. We identified the 20 companies in our dataset that acquired most of the biotech startups in the last five years and calculated the percentage of acquired companies developing orphan drugs (Fig. 4b). The biggest acquirer was Pfizer, followed by Gilead, Roche and Shire. Remarkably, neither Novartis, GlaxoSmithKline nor Roche acquired a company with an ODN on their lead compound in 2008-2012. This is in sharp contrast with the many ODNs assigned to drugs developed by these three companies in that same period.

Another surprise is the absence of three of the top pharmaceutical companies, AbbVie, Merck and Bayer, in the list of active acquirers. Shire, Celgene and Biomarin - all well-known for their interest in drug development for rare diseases-placed in the top 15 active acquirers and acquired almost solely companies with an ODN. Bristol-Myers Squibb lands in the top five, showing a keen interest in companies with an orphan designation, though it has a limited number of assigned ODNs itself. This analysis reveals that, although most pharmaceutical companies have in-house ODN programs, only a subset of big pharma is interested in acquiring orphan-designated drugs. For bioentrepreneurs looking to start a new venture in the orphan drug space, it will be important to develop strong inroads into this pharmaceutical subset, generate their input on development strategy early and closely follow their interests ${ }^{6}$.

\section{Conclusion}

Starting a company with the aim to develop drugs requires dedication, focus and a significant investment of capital. For bioentrepreneurs (and their venture capitalist investors), it is important to know whether these investments will pay off at the end. Our analysis, which a

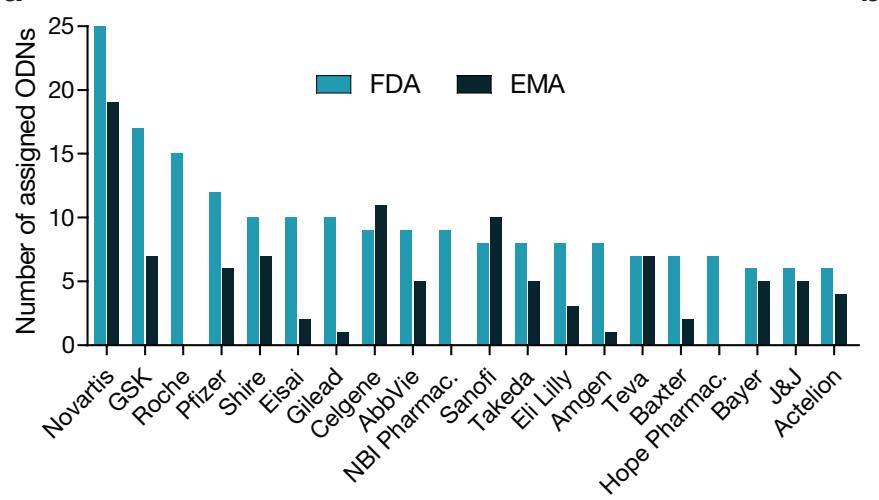

b

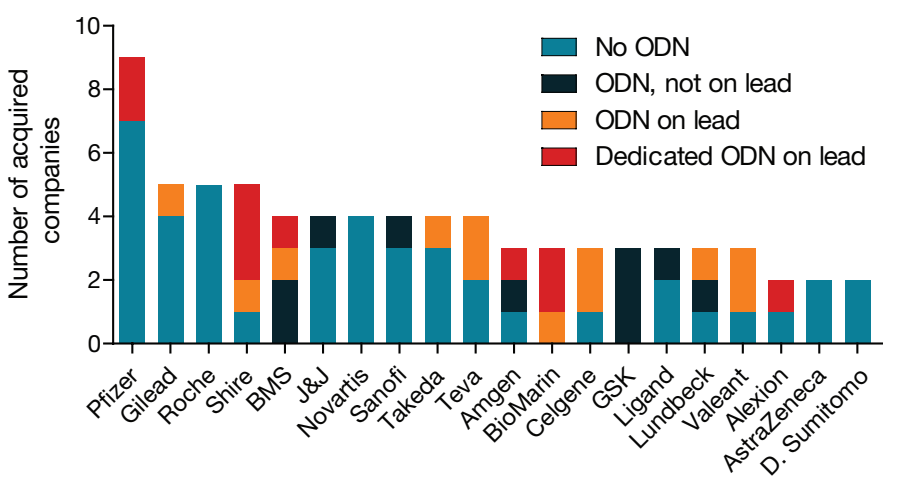

Figure 4 Most active companies and those with the most orphan designations (ODNs). (a) Bar graph of 20 companies that received the most ODNs from the US Food and Drug Administration (FDA), 2008-2012. Shown are data from both the FDA and the European Medicines Agency (EMA). AstraZeneca. AstraZeneca + Medlmmune; Celgene: Celgene + Abraxis; Eisai: Eisai + Morphotek; Eli Lilly: Eli Lilly + ImClone; GSK: GlaxoSmithKline + Stiefel; J\&J: Johnson \& Johnson + Janssen + Centocor; Novartis: Novartis + Alcon; Pfizer: Pfizer + Wyeth + Pharmacia + King; Roche: Roche + Genentech; Sanofi: Sanofi + Genzyme; Takeda: Takeda + Millennium; Teva: Teva + Cephalon; Valeant: Valeant + Biovail. (b) Bar graph displaying 20 most active acquirers in our dataset. 
focused on M\&A in 2008-2012, indicates that-from a financial point of view-no large differences exist between the development of orphan and non-orphan drugs. Development of orphan drugs is cheaper and faster, but this benefit is offset by acquisition at a later stage. A decision to invest or start a venture in orphan drug development should-as with drugs for common diseases-be driven mainly by assessments of risk and chance of success. Here it should be noted that we have not looked at attrition rates for orphan and non-orphan drug development, as it is virtually impossible to obtain data on drug development ongoing in all privately held companies. However, attrition rates would clearly have an important impact on the return of capital invested. Another important consideration is pricing of orphan drugsa subject of continuous debate ${ }^{5,7}$. Although high reimbursement prices are accepted for now, this may change in the future and could negatively impact deal terms. This may especially hold true for compounds that have limited clinical benefit or are not disease modifying.

Our analysis also reveals that despite a broad interest from big pharma in obtaining ODNs, only a limited set of players acquires rare disease-focused companies. Rare diseasefocused bioentrepreneurs should therefore keep a close watch on the wish list of this limited group and seek a strategic fit ${ }^{6}$. To further enhance their success rate, these bioentrepreneurs should include regulatory advice and engage with the authorities as early as possible, thereby laying a clear path toward final success.

\section{COMPETING FINANCIAL INTERESTS}

The authors declare competing financial interests: details are available in the online version of the paper (doi:/10.1038/nbt.2836).
1. Meekings, K.N., Williams, C.S. \& Arrowsmith, J.E. Orphan drug development: an economically viable strategy for biopharma R\&D. Drug Discov. Today 17 660-664 (2012)

2. Sasinowski, F.J. Quantum of effectiveness evidence in FDA's approval of orphan drugs. Drug Inf. J. 46 , 238-263 (2012)

3. Mullard, A. 2012 FDA drug approvals. Nat. Rev. Drug Discov. 12, 87-90 (2013).

4. Novartis Institutes for BioMedical Research. Proofof-concept: innovation in Novartis R\&D Novartis Institutes for BioMedical Research http://www. nibr.com/cs/groups/public/@nibr_com/documents/ document/n_prod_200338.pdf (March 2009).

5. Wellman-Labadie, O. \& Zhou, Y. The US Orphan Drug Act: rare disease research stimulator or commercial opportunity? Health Policy 95, 216-228 (2010)

6. Giniatullina, A., Boorsma, M., Mulder, G.-J. \& van Deventer, S. Building for big pharma. Nat. Biotechnol. 31, 284-287 (2013).

7. O'Sullivan, B.P., Orenstein, D.M. \& Milla, C.E. Pricing for orphan drugs: will the market bear what society cannot? JAMA 310, 1343-1344 (2013).

8. HBM Partners. HBM Pharma/Biotech M\&A Report 2012. HBM Partners http://www.hbmpartners.com/ wAssets/docs/industry-reports/HBM-Pharma-BiotechM-A-Report-2012.pdf (January 2013).

\section{Startups on the menu}

In 2009, Stanford University's Robert Malenka and Thomas Südhof gave a presentation at the San Francisco SciCafé proposing the use of short hairpin RNAs as a means to more rapidly identify the role of synaptic proteins in disease pathophysiology. Malenka pioneered the study of several proteins that alter the efficacy of neurotransmission across synapses, providing insights into the addictive effects of drugs, such as cocaine and amphetamines; Südhof's work on mechanisms for neurotransmitter release and the calcium-controlled fusion of synaptic vesicles in the presynaptic terminal was recently awarded a Nobel Prize. In 2010, Malenka and Südhof were among the cofounders of neurology startup Circuit Therapeutics, based in Menlo Park, California. Malenka talked to Nature Biotechnology about his experience.

\section{Nature Biotechnology: When did you first get} involved with Circuit Therapeutics?

Robert Malenka: I had been on the scientific advisory board for several biotechs and pharma firms over the previous 15 years and had looked at jobs in pharma, so for a while I was also considering founding my own company. One day I started brainstorming with my friend Karoly Nikolich, and it didn't take us too long to think about asking Karl Deisseroth and Tom Südhof to join. Karl had been my postdoc, and it was obvious that optogenetics had huge potential. Tom was a close scientific collaborator and was the world's leading molecular synaptic biologist-he'd just won the Nobel Prize. After some discussion, we additionally asked Scott Delp, chair of Stanford's Bioengineering Department, to join. That was our fivemember founding team. We were fortunate to find a wealthy angel investor who understood our ambitious vision, and we were off and running with further support from Stanford University.
NBT: How did you identify the programs in your lab most amenable for translation?

RM: As a clinical psychiatrist and a frequent advisor to industry, I was well aware of the challenges researchers faced in trying to develop novel approaches to drug discovery for brain disorders. My own lab's work was directly relevant, since we work on circuit and synaptic mechanisms that contribute to various forms of pathological experience-dependent plasticity, which are of direct relevance to many brain disorders. In many ways this is translated through my current role at Circuit as chair of the scientific advisory board, which allows me to meet with Circuit employees fairly frequently and have many informal e-mail conversations about the science and ways to take it forward.

\section{NBT: What lessons have you taken from your experience at Circuit?}

RM: I've learned that you never stop learning and need to be very facile in thinking about what your company might do and is doing. There's never too much feedback or advice; one must solicit input from many different, experienced [venture capitalists] and entrepreneurs with different types of backgrounds. At the same time, one needs to believe in one's own vision and intuitions. In retrospect, I think Circuit could have been a bit more disciplined in its early activities and tried to do too much. On the other hand, with a recent, successful series $B$ funding and external collaborations being seriously discussed, Circuit is in good shape with outstanding leadership. It will be very exciting to see where the company is two years from now. 